\title{
Prostodoncia parcial fija convencional, tecnología CADCAM - Zirconio y caracterizado gingival: Reporte de caso
}

\author{
Conventional fixed partial prosthodontics, CADCAM -Zirconio technology and \\ gingival characterization: Case report
}

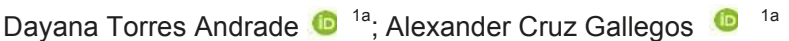

\section{RESUMEN}

La prostodoncia fija, se realiza en distintos materiales para sustituir estructuras dentales ausentes, que pueden ser totalmente metálicas o metálicas revestidas con cerámica, siendo generalmente muy utilizadas. En la actualidad, tenemos propuestas de prótesis fija con mejores resultados estéticos, como las prótesis libres de metal, que permiten al profesional reproducir mejor y con más detalle la anatomía dental y en este caso también el caracterizado gingival. Se dispone de materiales como el zirconio que tiene propiedades como la biocompatibilidad, baja conductividad térmica, resistencia a la corrosión, la naturalidad y la estética. Además, se tiene a disposición nuevos métodos tecnológicos con los cuales se puede lograr resultados muy satisfactorios, como la utilización de la tecnología CAD-CAM. El presente caso clínico está enfocado en la sustitución de órganos dentarios y tejido blando, para la rehabilitación del sector anterior, con materiales de características favorables, como el zirconio que contribuyan a devolver la funcionalidad, fonética, estética y masticación, mediante una prótesis fija de seis unidades libre de metal, con la mayor adaptabilidad tanto al tejido dental como gingival. Se verificó el avance de cada etapa clínica como la preparación de los pilares, colocación del provisional, impresiones, prueba de estructura y cementado.

Palabras claves: Rehabilitación bucal; Prótesis dental; Dentadura parcial fija; Diseño asistido por computadora; Circonio; Cerámica. (Fuente: DeCS BIREME)

\begin{abstract}
Fixed prosthodontics is performed in different materials to replace absent dental structures, which can be totally metallic or metallic coated with ceramic, being generally widely used. At present, we have proposals for fixed prostheses with better aesthetic results, such as metal-free prostheses, which allow the professional to reproduce better and in more detail the dental anatomy and in this case also the gingival character. Materials such as zirconia are available that have properties such as biocompatibility, low thermal conductivity, resistance to corrosion, naturalness and aesthetics. In addition, new technological methods are available with which very satisfactory results can be achieved, such as the use of CAD-CAM technology. The present clinical case is focused on the replacement of dental organs and soft tissue, for the rehabilitation of the anterior sector, with materials with favorable characteristics, such as zirconium that contribute to return functionality, phonetics, aesthetics and chewing, through a fixed prosthesis of six metal-free units, with the greatest adaptability to both dental and gingival tissue. The progress of each clinical stage was verified, such as the preparation of the abutments, placement of the provisional, impressions, test of the structure and cementation.
\end{abstract}

Keywords: Mouth Rehabilitation;Dental Prothesis; Dentadure, Partial, Fixed; Computer-Aided Design; Zirconium; Ceramics.(Source: MeSH NLM)

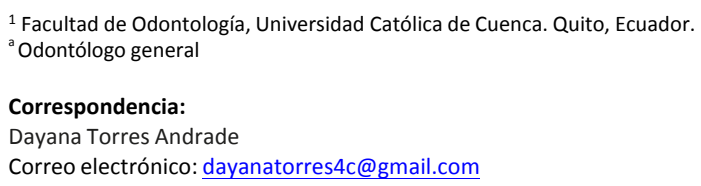

Este es un artículo Open Access distribuido bajo la licencia Creative Commons Atribución 4.0 Internacional (CC BY 4.0)

https://creativecommons.org/licenses/by/4.0/deed.es

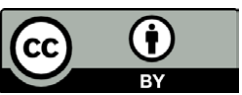

Citar como: Dayana Torres Andrade; Alexander Cruz Gallegos, Prostodoncia parcial fija convencional, tecnologíaCADCAM Zirconio y caracterizado gingival. KIRU. 2021;18(2):89-96. https://doi.org/10.24265/kiru.2021.v18n2.03. 


\section{INTRODUCCIÓN}

La tecnología junto a materiales cerámicos ha logrado grandes avances en odontología, el uso clínico de zirconio gracias a sus propiedades como la resistencia a flexión, a la fatiga y su alta tenacidad permite de manera amplia su aplicación en restauraciones dentales. En la actualidad, el zirconio se presenta en forma de Y-TZP que al oxidarse se convierte en una cerámica, compuesta por un $95 \%$ de óxido de circonio y un $5 \%$ de óxido de itrio. El diseño y fabricación delóxido de zirconio es asistido por un ordenador CAD/CAM este sistema creado desde 1980 se puede usar en el sillón, en un laboratorio dental o en un centro de fresado, mediante este se consigue una fabricación preestablecida, un método que permite crear de manera precisa su estructura para evitar las discrepancias marginales que pueden intervenir en la tasa de supervivencia de la prótesis, para evidenciar su calidad se recomienda medir las cofias antes del proceso de recubrimiento ya que en ocasiones el proceso de cocción de la porcelana puede intervenir en su ajuste ${ }^{(1-8)}$.

Los defectos óseos, provocan problemas estéticos en una futura restauración, Seibert 1983, describe al defecto clase III por ausencia vertical y horizontal de la brecha edéntula, como opción de tratamiento es el quirúrgico el cual demanda tiempo y un costo elevado. Por lo tanto, tenemos el desafío de rehabilitar con una prótesis fija más caracterizado gingival a través del diseño y fabricación asistida por computadora, que permitirá un resultado mucho más estético. Se debe considerar el equilibrio entre dientes y tejidos blandos faciales, al sonreír la mínima cantidad de exposición de encía se considera de 1 a $2 \mathrm{~mm}$ en pacientes jóvenes, para la determinación de la composición dental se toma en cuenta la relación existente entre ancho y alto de la corona de cada elemento dentario ${ }^{(9-16)}$.

Dentro de la a preparación de los dientes pilares se requiere una terminación de tipo chanfer $u$ hombro, ángulo de convergencia entre 6 y 12 grados, reducción vestibular, lingual y proximal es de $1.5 \mathrm{~mm}$, oclusal $1.5-2.0 \mathrm{~mm}$. Al referirnos a la cementación definitiva algunos resultados clínicos demuestran que el tipo de cemento utilizado no influye en la durabilidad de este tipo de rehabilitaciones por lo que se recomienda: fosfato de zinc, ionómero de vidrio ó cemento autoadhesivo ${ }^{(17-20)}$.

\section{REPORTE DEL CASO}

Paciente de 30 años de edad, de sexo femenino, sin antecedentes personales ni familiares optó por una prótesis fija de metal cerámica. Su motivo de consulta" la prótesis que uso me provoca inseguridad no puedo sonreír por tener estos dientes muy grandes y tampoco hablar cerca de las personas porque me huele mal la boca", siente rechazo quienes la rodean, su autoestima se ve afectada. Se realiza la odontograma, exámenes complementarios de imagen como lo es la radiografía panorámica, radiografías periapicales para valorar tejidos que circunda la porción apical de los pilares los cuales se encargan del soporte, reflejando un aspecto sano (Figura 1).

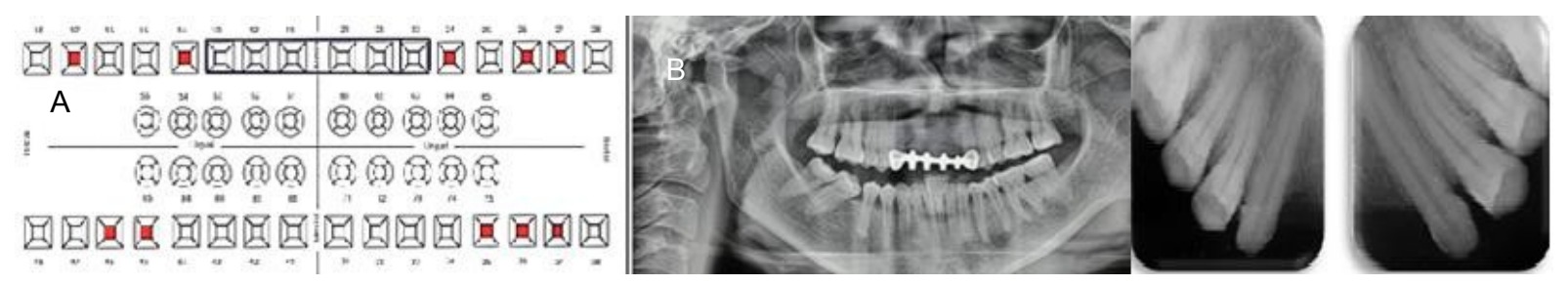

Figura 1. A. Registro digital del odontograma. B. Radiografía panorámica y radiografías periapicales piezas 13 y23, no se observa imágenes radiográficas compatibles con alguna patología 
Se realizó el registro fotográfico extraoral, se evidenció tejidos faciales normales y tercios simétricos. Al examen clínico intraoral se observó tejidos periodontales sin patología, una prótesis de metal - cerámica que reemplazaba las piezas $11,12,21,22$, como pilares, dientes vitales 13 y 23 y desadaptación de aproximadamente 2 a 3 $\mathrm{mm}$. entre el póntico y el reborde edéntulo. El sobrecontorneado, la desarmonía de la prótesis en relación a las piezas dentales de la paciente es evidente, además de la coloración grisácea típica de este material.
El plan de tratamiento tuvo como objetivo devolver la funcionalidad y equilibrio al sistema estomatognático y mejorar la estética del sector anterior. La terapia básica incluyó fisioterapia oral, operatoria dental, prótesis fija de zirconio dentosoportada para reponer las piezas $11,12,2122$ como pilares las piezas vitales 13 y 23. Se aplicó anestesia infiltrativa al paciente y se retiro la prótesis fija metal- porcelana, luego se le colocó un puente provisional de acrílico (Figura 3).

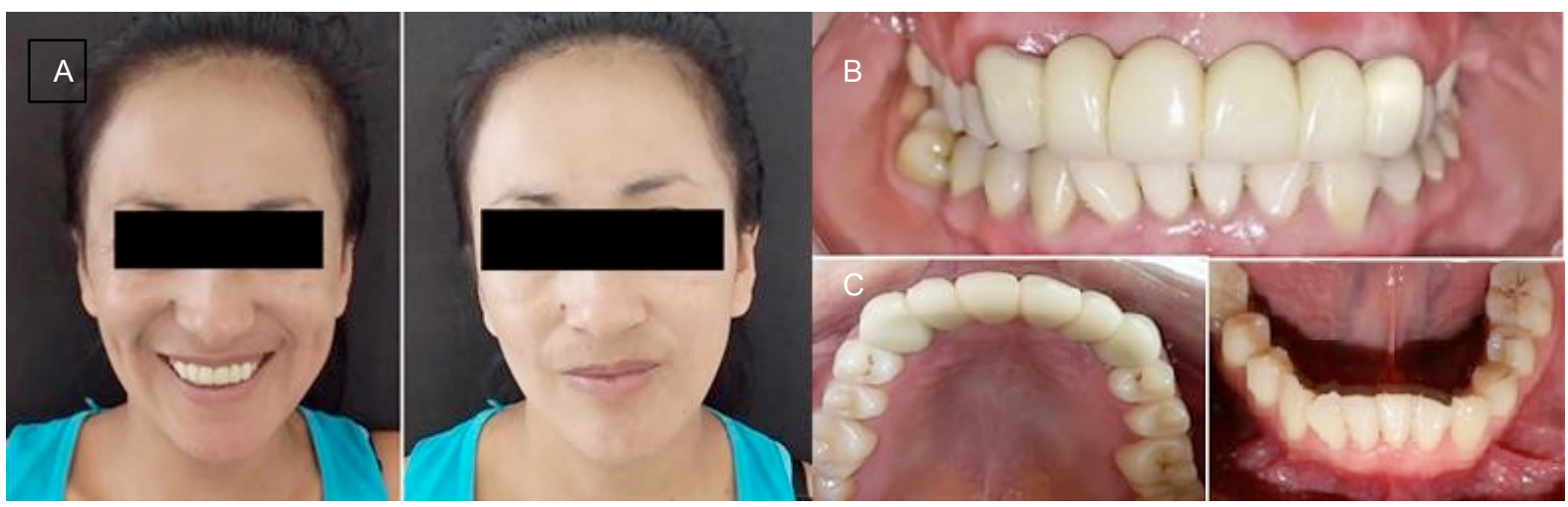

Figura 2. A. Vista inicial de la paciente con y sin sonrisa. B. Fotografía intraoral frontal que muestra la diferencia en las dimensiones de las piezas dentales de la prótesis fija en relación al resto de dientes. C. Vistas superior e inferior de las arcadas dentales.

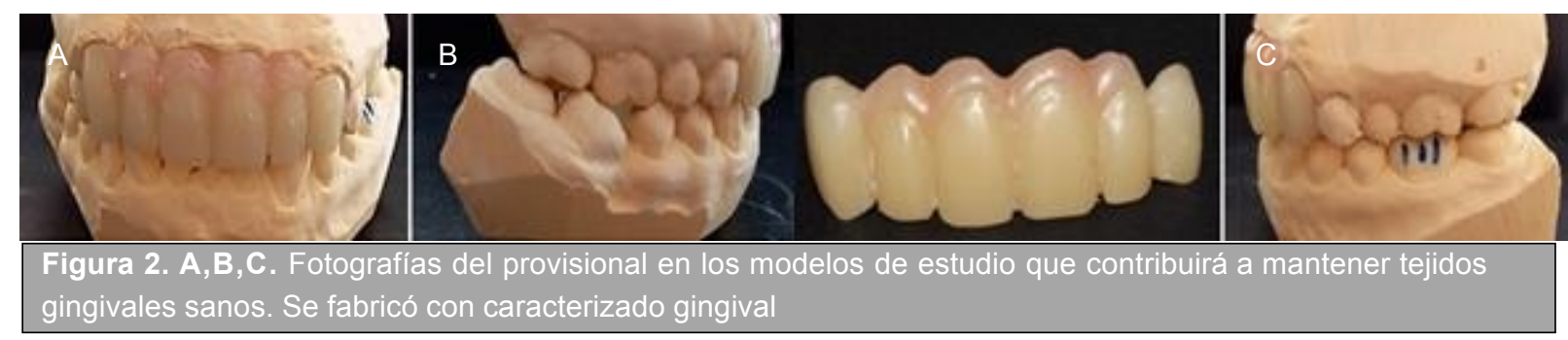

En otra cita se prepararon los pilares, para lo cual se utilizó una fresa tronco cónica. Una vez terminados los tallados, se colocó hilo retractor de 2 /0 previo a la impresión.
Se preparó la silicona de adhesión, con técnica a dos manos y se tomaron las impresiones definitivas (Figura 4). Se realizó la toma del color de dientes y encía (Figura 5). 


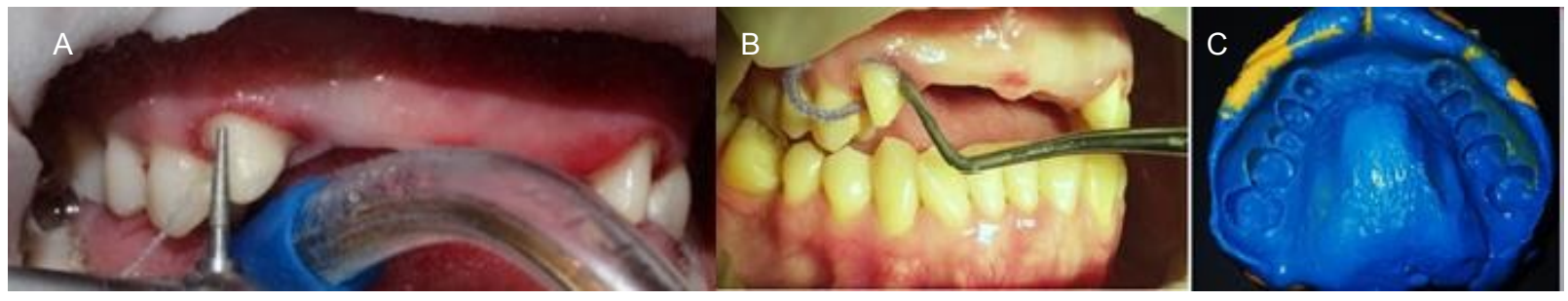

Figura 4. Preparación de los pilares, reducción oclusal 1.5- $2.0 \mathrm{~mm}$, reducción vestibular $1.5 \mathrm{~mm}$, terminación en chamfer. Colocación del hilo retractor. Toma de impresión definitiva con silicona de adición
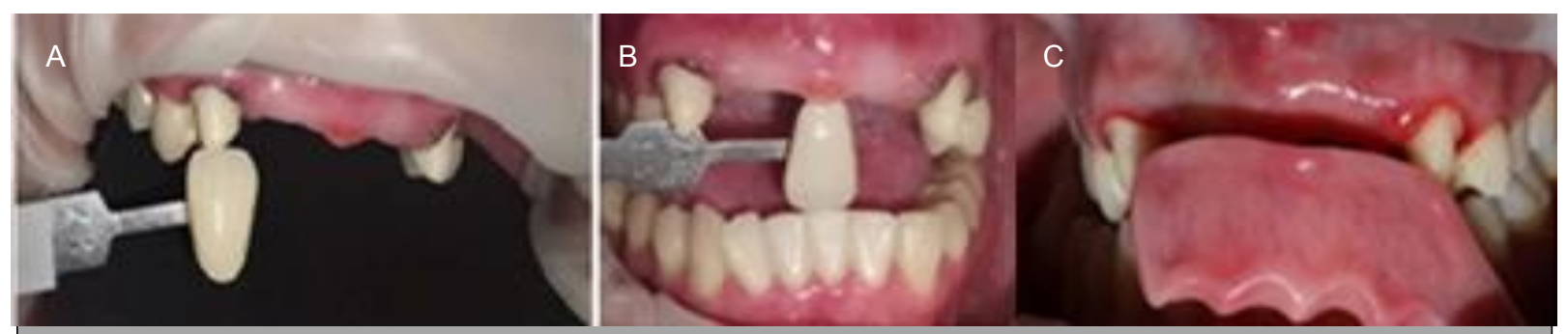

Figura 5. Toma de color de las piezas dentales y del tejido mucoso con la ayuda de un colorímetro de encía para un mejor resultado.

En la fase de laboratorio se diseñó la estructura de zirconio, la misma que fue evaluada en boca para corroborar el sellado marginal de la estructura y los pilares dentales. Obteniendo la adaptación deseada. En una próxima cita se probó la estructura cubierta de porcelana con el color de dientes y encía en base al registro de color (Figura 6).

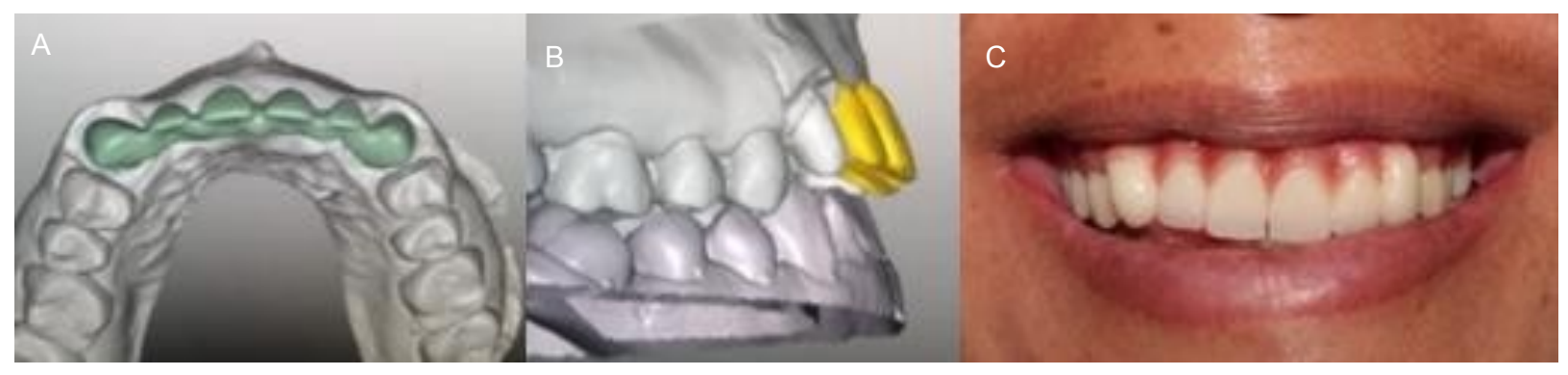


La fase final que se refiere al cementado de la prótesis, al evidenciar que los tejidos se encuentran en óptimas condiciones, se procede a la desinfección, arenado de la prótesis fija. Desinfección de los pilares con clorexidina y piedra pomex, preparación de iomómero de vidriode cementación definitiva del puente de zirconio, retiramos excesos (Figura 7).

A los 8 días de cementada la prótesis fija se realiza el control, se puede evidenciar el cambio en el autoestima de la paciente, en las fotografías finales intra y extraorales se registra mayor estética (Figura 8).
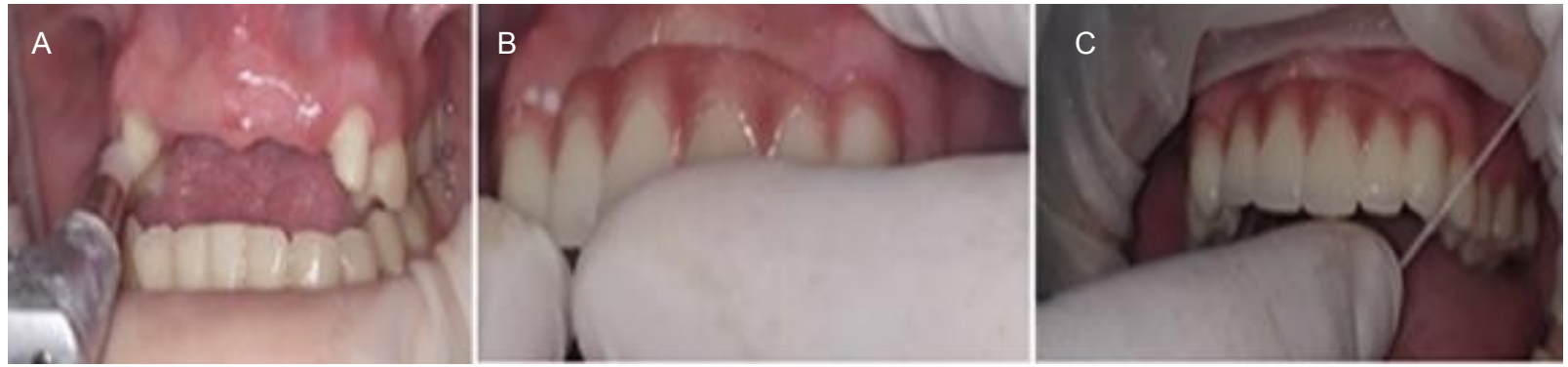

Figura 7. A. Pulido de los pilares previo a la cementación de la prótesis. B. Cementación de la prótesis. C. Remoción de excesos de cemento con hilo dental.

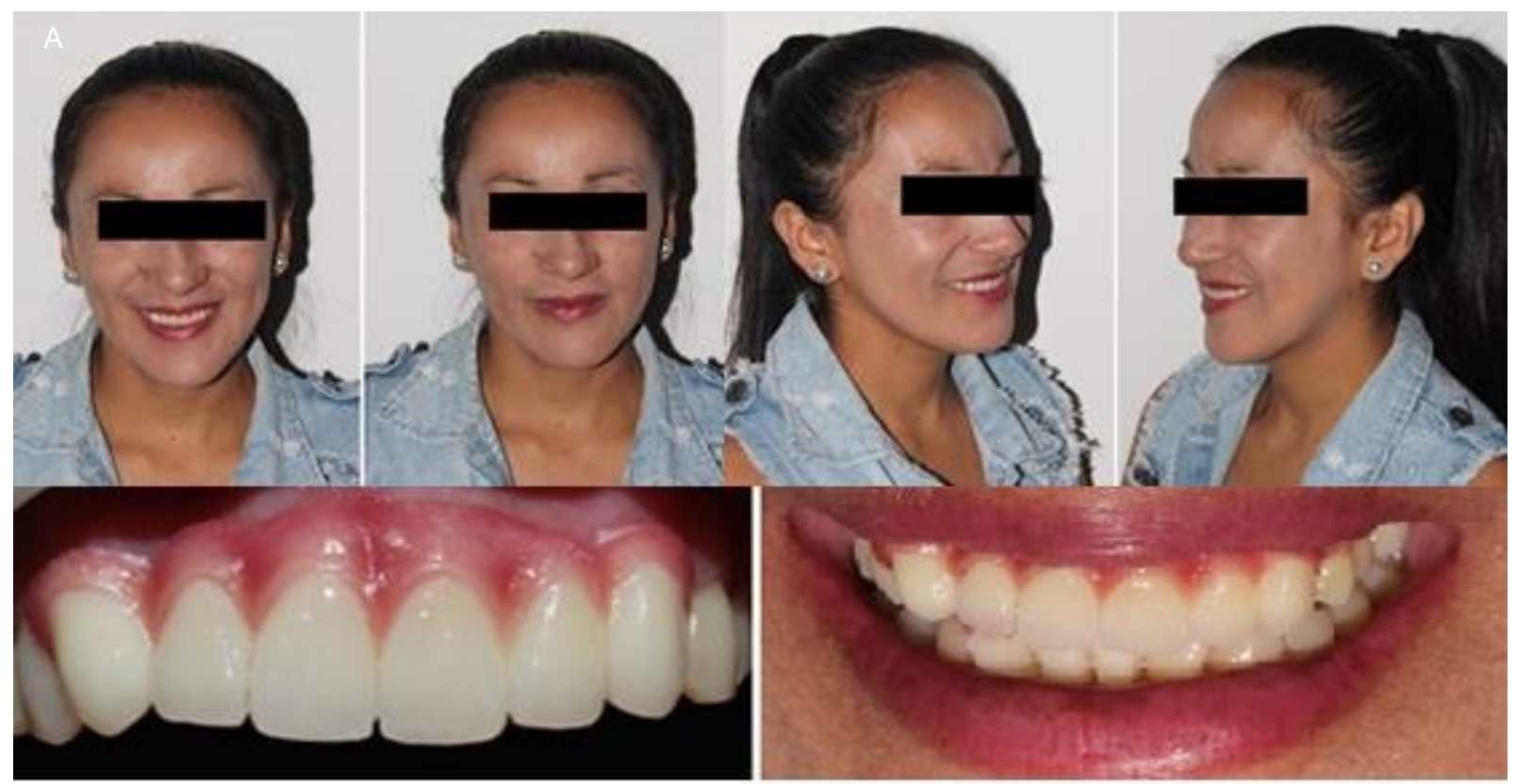




\section{DISCUSIÓN}

Se debe tomar en cuenta como método alternativo para la sustitución de defectos gingivales sin abordaje quirúrgico en el sector anterior a una restauración gingival protésica como parte de la rehabilitación, basándonos en un examen clínico y radiográfico para saber si el procedimiento para mejorar la estética blanca y rosa en realidad es viable, Nasrin y Jawad En su reporte de caso, usaron pautas para un asegurar la estética del paciente en donde el uso de porcelana de color gingival para reemplazar el volumen de tejido blando faltante cumplió con las expectativas del paciente, al ser un tratamiento no invasivo, con un costo y tiempo reducido para tan positivos resultados ${ }^{(21)}$.

Walton, en un estudio retrospectivo clínico evaluó 515 prótesis parciales fijas metal cerámica calculó una tasa de supervivencia de $96 \%$ en 5 años, $87 \%$ en 10 años y $85 \%$ en 15 años. Walton también reportó, que la principal causa de fracasos en este tipo de restauraciones fue fractura de dientes $38 \%$, compromiso periodontal $27 \%$, pérdida de retención $13 \%$, y caries $11 \%$.En cuanto al óxido de zircono, tiene propiedades mecánicas superiores y está indicada para restauraciones de piezas dentales anteriores, debido a la elevada opacidad del material, posee una alta resistencia a la flexión aproximada de 900 a 1200 Mpa y tenacidad de fractura de 9 a $10 \mathrm{Mpa} / \mathrm{mm}$, mayor biocompatibilidad, estudios revelan menor acumulación de bacterias alrededor de este material. Por lo tanto impacto positivo en la salud de los tejidos periodontales ${ }^{(22)}$.

Peter Pospiech reporta que según los resultados de ciertos estudios clínicos sobre prótesis fijas con estructura de zirconio la tasa chipping en la cerámica de recubrimiento (50\%) parece superar con la registrada con restauraciones de metalcerámica. Reuter y Brose reseñaron una tasa de astilla-miento del $2,5 \%$ en PPF de metalcerámica después de 5 años. Cuando se empleó como material de la estructura, cerámica infiltrada con vidrio, no se reseñó ningún astillamiento de la cerámica de recubrimiento después de 5 años" Sin embargo, Sailer et al. 2009, Schley et al. 2010, Beuer et al. Defiende que a frecuencia de chipping en prótesis fija de óxido de zirconio sobre dientes puede alcanzar el $25 \%$ a los $3-5$ años ${ }^{(23-24)}$.

Una ventaja del uso del zirconio es que, al ser fabricada con tecnología CAD-CAM se consigue mayor ajuste marginal, mayor adaptabilidad de la prótesis al tejido dental. Además a diferencia de las prótesis convencionales metal - cerámica, esta no refleja la traslucidez grisácea, por lo que resulta estético más aun cuando se trata de rehabilitar el sector anterior.

\section{CONCLUSIÓN}

En el presente caso, se obtuvo resultados satisfactorios al utilizar prótesis fija con estructura de zirconio, para reemplazar seis piezas dentales, en el sector antero superior. Además, gracias a esta tecnología se logró sustituir el tejido gingival con mayor naturalidad, armonía y estética. Finalmente es un proceso que puede evitar al paciente una intervención quirúrgica, ofrecer menor costo y elaborarse en menos tiempo.

Fuente de financiamiento: El estudio fue financiado por los autores.

Conflicto de intereses: Los autores declaran no tener conflictos de interés.

\section{REFERENCIAS BIBLIOGRÁFICAS}

1. Marcelo Cascante Calderón IVAISM. Cerámicas: una actualización. [Online].; 2019 [cited 2021 febrero 7]. Available from: https://revistadigital.uce.edu.ec/index.php/odontolo gia/article/view/2063/2157

2. Journal of International Oral Health. Evaluación del diseño asistido por computadora y las restauraciones de fabricación asistida por computadora: una revisión de la literatura. [Online].; 2015 [cited 2021 febrero 7]. Available from:

https://www.ncbi.nlm.nih.gov/pmc/articles/PMC440 9808/\#.

3. Jesús Peláez Rico CLSVRAMJS. Dossier. [Online].; 2016 [cited 2021 Febrero 11. Available from: $\quad$ https://www.sepes.org/wpcontent/uploads/difusion/gaceta-dental/DossierJesus-Pelaez-Rico.pdf

4. Avi Meirowitz YB,SL,EMyED. PubMed. [Online].; 2019 [cited 2021 Febrero 8. Available from: https://www.ncbi.nlm.nih.gov/pmc/articles/PMC656 7516/. 
5. Segura Andrés HVMJyAR. ¿circonio, cerámica o metal-porcelana? revisión de la literatura.. [Online].; 2014 [cited 2021 Febrero 8. Available from: $\quad:$ http://www.gacetadental.com/wp content/uploads/2014/05/259_CIENCIA_Circonio CEramicaPorcelana1.pdf.

6. Rubén Agustín-Panadero JLRR,AF,MFSR,yAFF. Circonia en prótesis fija. Una revisión de la literatura. J Clin Exp Dent. [Online].; 2014 [cited 2021 Febrero 8. Available from: https://www.ncbi.nlm.nih.gov/pmc/articles/PMC3.

7. Avi Meirowitz YB,SL,EM,yED. Una evaluación in vitro de coronas de circonio de ajuste marginal fabricadas por un laboratorio dental CAD-CAM y un centro de fresado. [Online].; 2019 [cited 2021 02 21. Available from: https://www.ncbi.nlm.nih.gov/pmc/articles/PMC65 67516/.

8. Matthias Rödiger LSySR. nfluencia de la selección del material en la precisión marginal de las cofias de corona simple de cerámica y metal fabricadas con CAD / CAM. [Online].; 2018 [cited 2021 febrero 21. Available from: https://www.ncbi.nlm.nih.gov/pmc/articles/PMC58 85340/.

9. García gargallo yGbM. Técnicas de preservación de alveolo y de aumento del reborde alveolar: Revisión de la literatura. avances en periodoncia. [Online].; 2013. [cited 2021 Febrero 9. Available from: Disponible http://scielo.isciii.es/pdf/peri/v28n2/original2.pdf.

10. Godoy C JECRLC. Carilla gingival: enmascara lo antiestético. [Online].; 2011 [cited 2021 febrero 11. Available from: https://www.ncbi.nlm.nih.gov/pmc/articles/PMC32 00029/.

11. Godoy C JECRLC. Aumento Tridimensional de un Reborde Alveolar Mediante. [Online].; 2008 [cited 2021 febrero 11. Available from: https://pdf.sciencedirectassets.com/311449/1s2.0-S0718539108X70016/1-s2.0S0718539108700065/main.pdf?X-Amz-Security Token=IQoJb3JpZ2luX2VjEL7\%2F\%2F\%2F\%2F \%2F\%2F\%2F\%2F\%2F\%2FwEaCXVzLWVhc3Qt MSJIMEYCIQCa4gS895c1dJWxWGBasNGL0k\% 2BwUbt8w\%2FalagpBQYpbBwlhAMR6eVBU.

12. Díez-Pérez $R$ CBXBMA. Cirugía plástica periodontal en dientes del sector. [Online].; 2016 [cited 2021 febrero 12. Available from: http://scielo.isciii.es/pdf/peri/v28n3/original4.pdf.

13. Londoño MA BP. LA SONRISA Y SUS DIMENSIONES. [Online]; 2012 [cited 2021 febrero 12 . Available from: http://www.scielo.org.co/pdf/rfoua/v23n2/v23n2a1 3.pdf.

14. Shipra Sepolia GS,RK,DKG,VJ,SCG. Visibilidad de la encía: un determinante importante para una sonrisa estética. [Online].; 2014 [cited 2021 febrero 12 . Available from:
https://www.ncbi.nlm.nih.gov/pmc/articles/PMC41 58592/.

15. Eugenio José García TmMdAOGJCGc. Aplicación clínica de los parámetros estéticos en odontología restauradora. Acta odontológica Venezolana disponible. [Online].; 2009 [cited 2021 Febrero 9. Available from: https://www.actaodontologica.com/ediciones/200 9/1/art-4/.

16. David Santiago Caceres Aguilar YLdASLCdMLFGSMCNEMVSLPM. Corrección de sonrisa gingival: del planeamiento digital a la técnica quirúrgica. [Online].; 2018 [cited 2021 febrero $12 . \quad$ Available from: https://www.actaodontologica.com/ediciones/201 8/2/art-3/.

17. Mallat E. Decálogo de uso del óxido de zirconio en prótesis fija. [Online].; 2016 [cited 2021 febrero $12 . \quad$ Available from: http://prosthodonticsmcm.com/decalogo-de-usodel-oxido-de-zirconio-en-protesis-fija/.

18. Osorio Vélez LS AMCRpsdcspr. AVANCES EN ODONTOESTOMATOLOGÍA. [Online].; 2018 [cited 2021 febrero 11. Available from: http://scielo.isciii.es/pdf/odonto/v25n5/original4.pd f.

19. Palomino DME, Rayo HG. CEMENTACIÓN DE ESTRUCTURAS PARA PRÓTESIS PARCIAL FIJA EN ZIRCONIA. [Online].; 2013 [cited 2021 febrero $11 . \quad$ Available from: http://www.scielo.org.co/scielo.php?script=sci_art text\&pid=S0121-246X2013000100011.

20. Yeimy Vargas Rodríguez MO. Protésica Fija: Puente Zirconio Sector Anterior Superior. [Online].; 2013 [cited 2021 febrero 11. Available from:

http://www.ulacit.ac.cr/files/revista/articulos/esp/r esumen/92 article6idental6.pdf.

21. Tair NRSyJAA. Reconstrucción protésica con agente cerámico de color gingival en restauración parcial fija en un paciente de 24 años. [Online].; 2012 [cited 2021 febrero 12. Available from: https://www.ncbi.nlm.nih.gov/pmc/articles/PMC36 52368/

22. Toro Gabrie NM. Estudio comparativo de una cerámica inyectada y una cerámica estratificada sobre el zirconio al ser sometido a fuerzas de cizalla. [Online].; 2011 [cited 2021 febrero 11. Available from: https://repository.ces.edu.co/bitstream/10946/108 8/1/Estudio_comparatico_ceramica.pdf.

23. Pospiech P. Chipping: ¿un problema inherente al sistema o derivado de la manipulación? [Online].; 2011. Available from: https://dialnet.unirioja.es/servlet/articulo?codigo= 3713782.

24. Viviane Gonçalves SBEBea. Efectos del espesor de la porcelana sobre la resistencia a la flexión y 
I

a propagación de grietas en un sistema de zirconia bicapa. [Online].; 2017 [cited 2021

febrero $12 . \quad$ Available from:

https://www.ncbi.nlm.nih.gov/pmc/articles/PMC58 06690/.

Dayana Torres Andrade

ORCID iD: (1) https://orcid.org/0000-0002-0836-1420

dayanatorres4c@gmail.com

Alexander Cruz Gallegos

ORCID iD: (1) https://orcid.org/0000-0002-6527-3793

alexandercruz1993@hotmail.com

Copyright $@$ La revista. La revista Kiru es publicada por la Facultad de Odontología de la Universidad de San Martín de Porres, en Lima, Perú. 